\title{
Analytical multi-soliton solutions of a (2+1)-dimensional breaking soliton equation
}

\author{
Shao-fu Wang*
}

\author{
*Correspondence: \\ nctsg09@163.com \\ College of Electrical \\ and Electronic Engineering, \\ Department of Electronics, \\ Anhui Science \\ and Technology University, \\ Bengbu, Fengyang 233100 \\ Anhui, People's Republic \\ of China
}

\begin{abstract}
The analytical solutions for a $(2+1)$-dimensional breaking solution equation is proposed in this paper by using mapping and projective method darboux transformation, and Some exact propagating solutions are constructed for this Breaking equation, and the $M \times N$ multi-soliton could be obtained by using Weierstrassp function and setting the perfect parameters. The potential application of breaking Soliton equation will be of great interest in future research.
\end{abstract}

Keywords: Breaking equation, Darboux transformation, Weierstrassp function, Multi-soliton

\section{Background}

In recent years, many research have studied soliton and its evolvements in nonlinear equations via kinds of method (Zarmi 2014; Chen and Ma 2013; Ashorman 2014; Zhang and Chen 2016; Meng and Gao 2014; Mohamed 2016; Liu and Liu 2016; Jiang and Ma 2012; Guo and Hao 2013; Dou et al. 2007). Vertex dynamics in multi-soliton solutions and some new exact solution of breaking equation are studied in Zarmi (2014) and Chen and Ma (2013), the methods of Multi-soliton Solutions are given in Ashorman (2014), Zhang and Chen (2016), Meng and Gao (2014), Mohamed (2016), Liu and Liu (2016), Jiang and Ma (2012), Guo and Hao (2013), Dou et al. (2007), Zuo and Gao (2014), Huang (2013), Liu and Luo (2013), Côtea and Muñoza (2014), Xu and Chen (2014), Hua and Chen (2014) and Zhang and Cai (2014), complex solutions for the [BLP System are proposed in $\mathrm{Ma}$ and $\mathrm{Xu}(2014)]$, and these so-called new solutions is identical to the universal formula in Doungmo Goufo (2016), Atangana and Doungmo Goufo (2015), Gao (2015a, b, c, d), Xie and Tian (2015), Sun and Tian (2015) and Zhen et al. (2015).

The aim of this paper is to investigate the analytical solutions of the $(2+1)$-dimensional breaking equation by the mapping and Darboux transformation method. And the dynamical behaviours of $(2+1)$-dimensional breaking equation will be discussed in detail.

The structure of this paper is as follows: In second section, the $(2+1)$ dimensional breaking equation is studied and its exact solutions are derived. And properties of this breaking equation will be investigated. In third section, influence of the parameters 
which are related to the analytical solution will also be discussed. Finally, the conclusion is drawn in fourth section.

\section{(2+1)-dimensional breaking equation and exact solution}

Consider (2+1)-dimensional breaking equation as follows (Dou et al. 2007):

$$
u_{t}+b u_{x x y}+4 b v_{x}+4 b u_{x} v=0
$$

In which, the functions of $u(x, y, t)$ and $v(x, y, t)$ are corresponding physical fields and set

$$
u_{y}=v_{x}
$$

In which $\mathrm{b}$ is the arbitrary function, substituting Eq. (2) into Eq. (1), the analytical solution of Eq. (1) could be gotten as follows:

$$
\begin{aligned}
& \left\{\begin{array}{l}
u=\frac{3}{2}(\ln f)_{x x}+u_{0} \\
v=\frac{3}{2}(\ln f)_{x y}+v_{0}
\end{array}\right. \\
& f=a_{0}+a_{1} p(x)+a_{2} q(y, t)+a_{3} p(x) q(y, t)
\end{aligned}
$$

In which, $u(x, y, t)$ is seed solution of equation, $u_{0}=a(x), v_{0}=0$, the parameters $a_{0}, a_{1}, a_{2}, a_{3}$ are constants.

Substituting Eqs. (3) and (4) into Eq. (1), the analytical solution of (2+1) dimensional breaking equation could be gotten as follows (Dou et al. 2007)

$$
\begin{aligned}
& u=-\frac{3}{2}\left[\frac{\left(a_{1}+a_{3} q\right)^{2} p_{x}^{2}}{\left(a_{0}+a_{1} p+a_{2} q+a_{3} p q\right)^{2}}-\frac{\left(a_{1}+a_{3} q\right) p_{x x}}{\left(a_{0}+a_{1} p+a_{2} q+a_{3} p q\right)}+\frac{b f_{x x x}+c f_{x}}{6 b f_{x}}\right] \\
& v=\frac{3}{2}\left[\frac{\left(a_{3} a_{0}-a_{1} a_{2}\right) p_{x} q_{y}}{\left(a_{0}+a_{1} p+a_{2} q+a_{3} p q\right)^{2}}\right]
\end{aligned}
$$

\section{$M \times N$ multi-soliton}

Define the Weierstrassp function as following

$$
\begin{aligned}
& X_{n+1}=X_{n}+a^{n} \cos \left(2 \pi b^{k} x\right) \\
& Y_{n+1}=Y_{n}+a^{n} \cos \left(2 \pi b^{n} y\right)
\end{aligned}
$$

In which $0<a<1, a b \geq 1$, and when the parameters $a=0.5, b=3, n=10$, its response is shown in Fig. 1 and we set

$$
\begin{aligned}
& \phi(x)=X_{n+1} ; \\
& \varphi(y)=Y_{n+1} ;
\end{aligned}
$$

when $p=\phi\left(k x, k_{2}, k_{3}\right), q=\varphi\left(y+c t, k_{2}, k_{3}\right)$, The Eq. (7) can be given as

$$
v=\frac{3}{2}\left[\frac{\left(a_{3} a_{0}-a_{1} a_{2}\right) \phi_{x} \varphi_{y}}{\left(a_{0}+a_{1} \varphi+a_{2} \phi+a_{3} \phi \varphi\right)^{2}}\right]
$$

in which

$$
\phi_{x}=\sqrt{4 \phi^{3}-k_{2} \phi-k_{3}}
$$




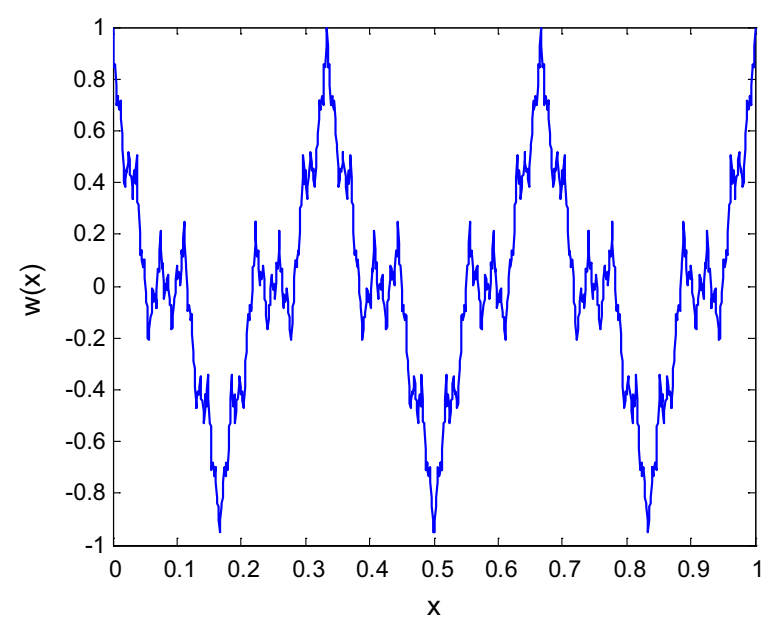

Fig. 1 The response of Weierstrassp function

$$
\varphi_{y}=\sqrt{4 \varphi^{3}-k_{2} \varphi-k_{3}}
$$

When the parameters $k, c, k_{2}, k_{3}, n$ are selected as different constants, the $N \times M$ multi-soliton could be achieved according to Eq. (11), when the parameters $a_{0}=4 ; a_{1}=1 ; a_{2}=1 ; a_{3}=1 ; k=0.6 ; k_{2}=1 ; k_{3}=1 ; c=1 ; n=5$, and $x \in[-3,3], y \in[-3,3]$, the $5 \times 6$ multi-soliton are shown in Fig. 2, and $x \in[-5,5], y \in[-5,5]$, the $10 \times 10$ multi-soliton structure are shown in Fig. 3 .

From Figs. 2 and 3, it can be seen that multi-soliton could be obtained by selecting the Weierstrassp function.

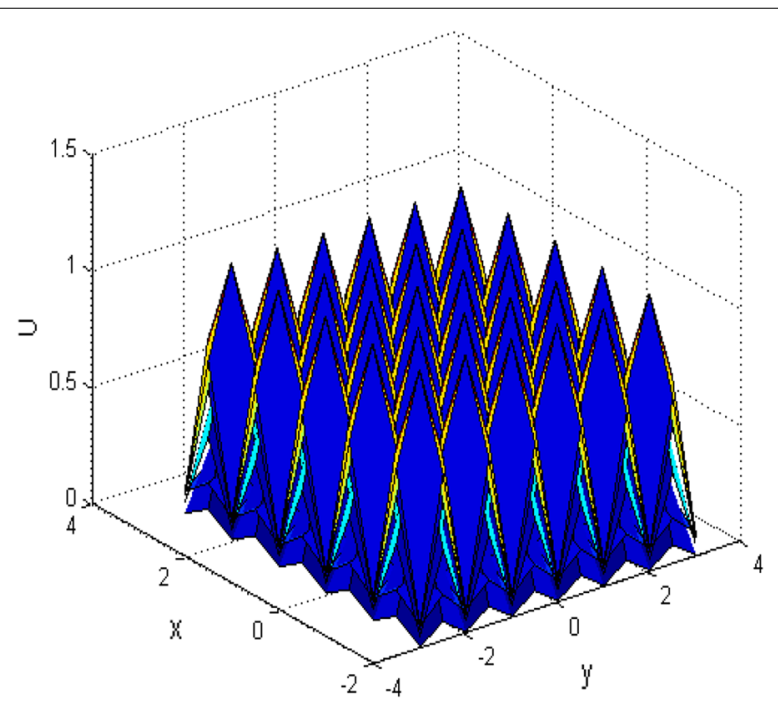

Fig. 2 The $5 \times 6$ multi soliton structure 


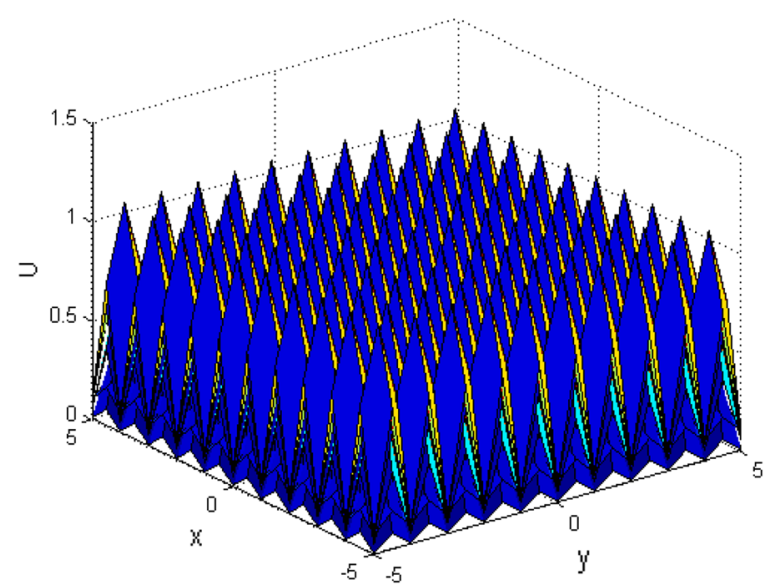

Fig. $310 \times 10$ multi soliton structure

\section{Conclusion}

To summarize, we have constructed $M \times N$ multi-solutions of a (2+1)-dimensional breaking equation using Darboux transformation and mapping method. The analytical solutions which changes the shape of the solutions are explored and the derived analytical expressions of $(2+1)$-dimensional breaking equation can be used in communication system, and it is highly anticipated that this investigation on $(2+1)$-dimensional breaking equation may have wider application in various physical models.

\section{Competing interests}

The authors declare that they have no competing interests.

Received: 11 April 2016 Accepted: 24 May 2016

Published online: 27 June 2016

\section{References}

Ashorman R (2014) Multi-soliton solutions for a class of fifth-order evolution equations. Int J Hybrid Inf Technol 7(4):11-18 Atangana A, Doungmo Goufo EF (2015) A model of the groundwater flowing within a leaky aquifer using the concept of local variable order derivative. J Nonlinear Sci Appl 8(5):763-775

Chen Y-M, Ma S-H (2013) New exact solution of a (3+1)-dimensional Jimbo-Miwa system. Chin Phys B 22:5101-5105

Côtea R, Muñoza C (2014) Multi-solitons for nonlinear Klein-Gordon equations. Forum Math Sigma 2:e15

Dou F-Q, Sun J-A et al (2007) The new periodic wave solutions and localized excitations for (2+1)-dimensional Breaking Soliton equation. J Northwest Norm Univ 43(1):34-38

Doungmo Goufo EF (2016) Application of the caputo-fabrizio fractional derivative without singular Kernel to Kortewegde Vries-Bergers equation. Math Model Anal 21(2):188-198

Gao X-Y (2015a) Comment on "Solitons, Bäcklund transformation, and Lax pair for the (2+1)-dimensional Boiti-LeonPempinelli equation for the water waves". J Math Phys 56(1):014101

Gao X-Y (2015b) Bäcklund transformation and shock-wave-type solutions for a generalized (3+1)-dimensional variablecoefficient B-type Kadomtsev-Petviashvili equation in fluid mechanics. Ocean Eng 96:245-247

Gao X-Y (2015c) Incompressible-fluid symbolic computation and bäcklund transformation: (3+1)-dimensional variablecoefficient Boiti-Leon-Manna-Pempinelli model. Z Naturforschung A 70(1):59-61

Gao X-Y (2015d) Variety of the cosmic plasmas: general variable-coefficient Korteweg-de Vries-Burgers equation with experimental/observational support. Europhys Lett 110(1):15002-15007

Guo R, Hao H-Q (2013) Breathers and multi-soliton solutions for the higher-order generalized nonlinear Schrödinger equation. Commun Nonlinear Sci Numer Simul 18(9):2426-2435

Hua Y-J, Chen H-L (2014) New kink multi-soliton solutions for the (3+1)-dimensional potential-Yu-Toda-Sasa-Fukuyama equation. Appl Math Comput 234:548-556

Huang Y (2013) Explicit multi-soliton solutions for the KdV equation by Darboux transformation. Int J Appl Math 43(3):135-137

Jiang L-H, Ma S-H (2012) New soliton solutions and soliton evolvements for the (3+1)-dimensional Burgers system. Acta Phys Sin 61:0510-0514 
Liu N, Liu Y-S (2016) New multi-soliton solutions of a (3+1)-dimensional nonlinear evolution equation. Comput Math Appl 71(8):1645-1654

Liu J, Luo H-Y (2013) New multi-soliton solutions for generalized burgers-huxley equation. Therm Sci 17(5):1486-1489

Ma S-H, Xu G-H (2014) Complex solutions and novel complex wave localized excitations for the (2+1)-dimenaional BoitiLeon-Pempinelli System. Chin Phys B 23:0511-0515

Meng G-Q, Gao Y-T (2014) Multi-soliton and double Wronskian solutions of a (2+1)-dimensional modified Heisenberg ferromagnetic system. Comput Math Appl 66(12):2559-2569

Mohamed S (2016) Osman Multi-soliton rational solutions for some nonlinear evolution equations. Open Phys 14(1):26-36

Sun W-R, Tian B (2015) Nonautonomous matter-wave solitons in a Bose-Einstein condensate with an external potential. J Phys Soc Jpn 84:074003

Xie X-Y, Tian B (2015) Solitary wave and multi-front wave collisions for the BKP equation in physics, biology and electrical networks. Mod Phys Lett B 29:1550192

Xu Z-H, Chen H-L (2014) Resonance and deflection of multi-soliton to the (2+1)-dimensional Kadomtsev-Petviashvili equation. Nonlinear Dyn 78:461-466

Zarmi Y (2014) Vertex dynamics in multi-soliton solutions of Kadomtsev-Petviashvili II equation. Nonlinear Sci 27:1499-1523

Zhang S, Cai B (2014) Multi-soliton solutions of a variable-coefficient KdV hierarchy. Nonlinear Dyn 78(3):1593-1600

Zhang C-C, Chen A-H (2016) Bilinear form and new multi-soliton solutions of the classical Boussinesq-Burgers system. Appl Math Lett 58:133-139

Zhen H-L, Tian B et al (2015) Soliton solutions and chaotic motions of the Zakharov equations for the Langmuir wave in the plasma. Phys Plasmas 22(3):1-9

Zuo D-W, Gao Y-T (2014) Multi-soliton solutions for the three-coupled KdV equations engendered by the Neumann system. Nonlinear Dyn 75(4):701-708

\section{Submit your manuscript to a SpringerOpen ${ }^{\circ}$ journal and benefit from:}

- Convenient online submission

\section{- Rigorous peer review}

- Immediate publication on acceptance

- Open access: articles freely available online

- High visibility within the field

- Retaining the copyright to your article

Submit your next manuscript at $\boldsymbol{\nabla}$ springeropen.com 